\title{
LIGHT SIDE OF COMPACTNESS IN LEBESGUE SPACES: SUDAKOV THEOREM
}

\author{
Przemysław Górka and Humberto Rafeiro \\ Warsaw University of Technology, Department of Mathematics and Information Sciences \\ Ul. Koszykowa 75, 00-662 Warsaw, Poland; p.gorka@mini.pw.edu.pl \\ Pontificia Universidad Javeriana, Facultad de Ciencias, Departamento de Matemáticas \\ Cra. 7 No. 43-82, Bogotá, Colombia; silva-h@javeriana.edu.co
}

\begin{abstract}
In this note we show that, in the case of bounded sets in metric spaces with some additional structure, the boundedness of a family of Lebesgue $p$-summable functions follow from a certain uniform limit norm condition. As a byproduct, the well known Riesz-Kolmogorov compactness theorem can be formulated only with one condition.
\end{abstract}

\section{Introduction}

The classical theorem of Kolmogorov [11], sometimes also called Riesz-Kolmogorov theorem, characterizes the compactness of sets of functions in Lebesgue spaces. In the original formulation of Kolmogorov the theorem is the following:

Theorem 1.1. (Kolmogorov) Suppose $\mathcal{F}$ is a set of functions in $L^{p}([0,1])(1<$ $p<\infty)$. In order that this set be relatively compact, it is necessary and sufficient that both of the following conditions be satisfied:

(K1) the set $\mathcal{F}$ is bounded in $L^{p}$;

(K2) $\lim _{h \rightarrow 0}\left\|f_{h}-f\right\|_{p}=0$ uniformly with respect to $f \in \mathcal{F}$, where $f_{h}$ denotes the well-known Steklov function, viz.

$$
f_{h}(x)=\frac{1}{2 h} \int_{x-h}^{x+h} f(t) d t .
$$

After that, Tamarkin [18] extended the result to the case where the underlying space can be unbounded, with an additional condition related to the behaviour at infinity. Tulajkov [19] showed that Tamarkin's result was true even when $p=1$. Finally, Sudakov [16] showed that condition (K1) follows from condition (K2). All the previous results were proved in the framework of one dimensional Euclidean space.

The Riesz-Kolmogorov compactness theorem has also been extended to other function spaces, for example, it was extended by Takahashi [17] for Orlicz spaces satisfying the $\Delta_{2}$-condition, by Goes and Welland [2] for continuously regular Köthe spaces, by Musielak [12] to Musielak-Orlicz spaces, by Rafeiro [14] to variable exponent Lebesgue spaces, by Rafeiro and Vargas [15] to grand Lebesgue spaces, by Górka and Rafeiro [8] to grand variable Lebesgue spaces, by Górka and Macios [6, 7] to Lebesgue spaces in metric measure spaces, just to name a few. Weil [20] showed the compactness theorem in $L^{p}(G)$, where $G$ is a locally compact group. Pego [13] (see [4] and [5]) formulated Kolmogorov theorem for $p=2$ in terms of the Fourier transform.

https://doi.org/10.5186/aasfm.2017.4209

2010 Mathematics Subject Classification: Primary 46B50; Secondary 46E30.

Key words: Compactness, Riesz-Kolmogorov theorem, metric measure spaces. 
For a more detailed account regarding the history of the Riesz-Kolmogorov theorem, see [9].

In this small note we want to show that, whenever we are working in the general framework of metric measure spaces, condition (K1) is superfluous since it is a consequence of condition (K2).

\section{Preliminaries}

We shall denote the average of locally integrable function $f$ over the measurable set $A$ in the following manner

$$
(f)_{A}=f_{A} f d \mu=\frac{1}{\mu(A)} \int_{A} f d \mu .
$$

Let $(X, \rho, \mu)$ be a metric measure space equipped with a metric $\rho$ and a Borel regular measure $\mu$. We assume throughout the paper that the measure of every open nonempty set is positive and that the measure of every bounded set is finite. Additionally, we assume that the measure $\mu$ satisfies a doubling condition. This means that, there exists a constant $C_{d}>0$ such that for every ball $B(x, r)$,

$$
\mu(B(x, 2 r)) \leq C_{d} \mu(B(x, r)) .
$$

Now, let us recall the notion of continuity of a measure with respect to a metric (see $[3,1])$.

Definition 2.1. Let $(X, \rho, \mu)$ be a metric measure space. The measure $\mu$ is said to be continuous with respect to the metric $\rho$ if for all $x \in X$ and $r>0$ the following condition holds:

$$
\lim _{y \rightarrow x} \mu(B(x, r) \Delta B(y, r))=0,
$$

where $A \Delta B$ stands for the symmetric difference, i.e. $A \Delta B:=A \backslash B \cup B \backslash A$.

For example, when $(X, \rho, \mu)$ is a geodesic space (cf. [10]) and the measure $\mu$ is doubling, then $\mu$ is continuous with respect to the metric $\rho$ (see [1]).

Now, we can recall the charecterization of relatively compact sets in $L^{p}(X, \rho, \mu)$ from [6].

Theorem 2.2. Let $(X, \rho, \mu)$ be a metric measure space and $1<p<\infty$. Suppose moreover, that there exists $\theta>0$ such that $\mu(B(x, 1)) \geq \theta$. Let $x_{0} \in X$, then the subset $\mathcal{F}$ of $L^{p}(X, \mu)$ is relatively compact in $L^{p}(X, \mu)$ if and only if the following conditions are satisfied:

$$
\begin{aligned}
& \mathcal{F} \text { is bounded, } \\
& \lim _{R \rightarrow \infty} \int_{X \backslash B\left(x_{0}, R\right)}|f(x)|^{p} d \mu(x)=0, \quad \text { uniformly for } f \in \mathcal{F}, \\
& \lim _{r \rightarrow 0} \int_{X}\left|f(x)-(f)_{B(x, r)}\right|^{p} d \mu(x)=0, \quad \text { uniformly for } f \in \mathcal{F} .
\end{aligned}
$$

\section{Main result}

The main result of this paper is the following.

Theorem 3.1. Assume that $(X, \rho, \mu)$ is a connected metric measure space with continuous measure satisfying the doubling condition. Suppose, moreover, that balls are relatively compact and there exists $\theta>0$ such that $\mu(B(x, 1)) \geq \theta$. Let $1<p<$ 
$\infty$ and $D$ be a bounded subset of $X$ such that $X \backslash \bar{D} \neq \emptyset$. If the family $\mathcal{F}$ in $L^{p}(D, \mu)$ satisfies

$$
\lim _{r \rightarrow 0} \int_{X}\left|f(x)-(f)_{B(x, r)}\right|^{p} d \mu(x)=0 \quad \text { uniformly for } f \in \mathcal{F},
$$

where we continue the function $f$ by zero beyond $D$, then $\mathcal{F}$ is bounded.

In order to prove Theorem 3.1 we will need some auxiliary results. We start with the following lemma.

Lemma 3.2. Let $h>0$ and denote by $\mathbf{1}_{D}$ the characteristic function of the set $D$. Then the operator $U: L^{p}(X) \longrightarrow L^{p}(X)$ given by

$$
U f(x)=f_{B(x, h)} \mathbf{1}_{D} f d \mu
$$

is compact.

Proof. Let us take $V=B(0,1) \subset L^{p}(X)$. We shall show that the set $U(V)$ is relatively compact in $L^{p}(X)$. For this purpose, we shall use the characterization of relatively compact sets in $L^{p}(X)$ from Theorem 2.2. Since $D$ is bounded, there exists a ball $B\left(x_{0}, r\right)$ such that $D \subset B\left(x_{0}, r\right)$. Thus, for $f \in V$ we have $\operatorname{supp}(U f) \subset$ $B\left(x_{0}, r+h\right)=: W_{h}$. Hence, by the Jensen inequality, we get

$$
\begin{aligned}
\|U f\|_{L^{p}(X)}^{p} & =\|U f\|_{L^{p}\left(W_{h}\right)}^{p}=\int_{W_{h}}|U f|^{p} d \mu=\int_{W_{h}}\left|f_{B(x, h)} \mathbf{1}_{D} f d \mu\right|^{p} d \mu \\
& \leq \int_{W_{h}} f_{B(x, h)}|f|^{p} d \mu d \mu \leq\|f\|_{L^{p}(X)}^{p} \frac{\mu\left(W_{h}\right)}{\inf _{x \in X} \mu(B(x, h))} .
\end{aligned}
$$

Since $\mu$ is doubling and $\mu(B(x, 1)) \geq \theta$, we have $\inf _{x \in X} \mu(B(x, h))>0$. Thus, we get that $U(V)$ is bounded. Moreover, since $\operatorname{supp}(U f) \subset W_{h}$, we get that $\|U f\|_{L^{p}\left(X \backslash W_{h}\right)}=$ 0 . Finally, it remains to show that the family $U(f)$ is uniformly $L^{p}$-equicontinuous. Let $h>r>0$. We have

$$
\begin{aligned}
& \int_{X}\left|U f(x)-f_{B(x, r)} U f(y) d \mu(y)\right|^{p} d \mu(x) \\
& =\int_{W_{2 h}}\left|f_{B(x, r)}\left(f_{B(x, h)} \mathbf{1}_{D} f(z) d \mu(z)-f_{B(y, h)} \mathbf{1}_{D} f(z) d \mu(z)\right) d \mu(y)\right|^{p} d \mu(x) .
\end{aligned}
$$

On the other hand, from the proof of Lemma 4.3 in [1] and by the Hölder inequality we have

$$
\begin{aligned}
& \left|f_{B(x, h)} \mathbf{1}_{D} f(z) d \mu(z)-f_{B(y, h)} \mathbf{1}_{D} f(z) d \mu(z)\right| \\
& \leq \frac{1}{\mu(B(x, h))} \int_{B(x, h) \Delta B(y, h)}\left|\mathbf{1}_{D} f(z)\right| d \mu(z)+\frac{\mu(B(x, h) \Delta B(y, h))}{\mu(B(x, h)) \mu(B(y, h))} \int_{B(y, h)}\left|\mathbf{1}_{D} f(z)\right| d \mu(z) \\
& \leq\|f\|_{L^{p}(X)}\left(\frac{\mu(B(x, h) \Delta B(y, h))^{1-1 / p}}{\mu(B(x, h))}+\frac{\mu(B(x, h) \Delta B(y, h))}{\mu(B(x, h)) \mu(B(y, h))^{1 / p}}\right) .
\end{aligned}
$$

Hence, we obtain

$$
\int_{X}\left|U f(x)-f_{B(x, r)} U f(y) d \mu(y)\right|^{p} d \mu(x) \leq\|f\|_{L^{p}(X)}^{p} \int_{W_{2 h}}|I(x)|^{p} d \mu(x) .
$$


where

$$
I(x)=f_{B(x, r)}\left(\frac{\mu(B(x, h) \Delta B(y, h))^{1-1 / p}}{\mu(B(x, h))}+\frac{\mu(B(x, h) \Delta B(y, h))}{\mu(B(x, h)) \mu(B(y, h))^{1 / p}}\right) d \mu(y)
$$

By virtue of Lebesgue differentiation theorem (see e.g., [10]) we have

$$
\lim _{r \rightarrow 0} f_{B(x, r)}\left(\frac{\mu(B(x, h) \Delta B(y, h))^{1-1 / p}}{\mu(B(x, h))}+\frac{\mu(B(x, h) \Delta B(y, h))}{\mu(B(x, h)) \mu(B(y, h))^{1 / p}}\right) d \mu(y)=0 .
$$

Furthermore,

$$
\begin{aligned}
& \left|f_{B(x, r)}\left(\frac{\mu(B(x, h) \Delta B(y, h))^{1-1 / p}}{\mu(B(x, h))}+\frac{\mu(B(x, h) \Delta B(y, h))}{\mu(B(x, h)) \mu(B(y, h))^{1 / p}}\right) d \mu(y)\right|^{p} \\
& \leq\left(\frac{\mu\left(W_{4 h}\right)^{1-1 / p}}{\inf _{x \in X} \mu(B(x, h))}+\frac{\mu\left(W_{4 h}\right)}{\inf _{x \in X} \mu(B(x, h))^{1+1 / p}}\right)^{p} .
\end{aligned}
$$

Finally, the Lebesgue theorem finishes the proof.

We will also need the following result.

Lemma 3.3. 1 is not an eigenvalue of $U$.

Proof. Let us take $f \in L^{p}(X)$ such that $U f=f$. We shall show that $f=0$. Since the measure $\mu$ is continuous, from the proof of the previous lemma we have $f \in C(X)$. Moreover, from the proof of the previous lemma we have that supp $f \subset$ $W_{h}=B\left(x_{0}, r+h\right)$. Next, let us take a ball $B$ such that $W_{h} \subset B$ and $\bar{D} \subset B$. Suppose that $M=\sup _{x \in \bar{B}} f(x)>0$ and let

$$
C=\{x \in X: f(x)=M\} .
$$

Next, let us take $x_{0} \in \partial C$. Due to the fact that $C$ is closed, we have that $B\left(x_{0}, h\right) \cap$ $(X \backslash C)$ is an open nonempty set. Thus $\mu\left((X \backslash C) \cap B\left(x_{0}, h\right)\right)>0$. This contradicts our assumption that $f\left(x_{0}\right)=U f\left(x_{0}\right)$.

We now prove the main result.

Proof of Theorem 3.1. For this purpose we use the Riesz-Schauder theory (see e.g., [21]). Since $U$ is compact and 1 is not an eigenvalue of $U$, we get that $(U-I)^{-1}$ is bounded. On the other hand, we have $\|U f-f\|_{L^{p}(X)} \leq C$ for $f \in \mathcal{F}$ and some positive constant $C$. Thus,

$$
\|f\|_{L^{p}(X)} \leq C\left\|(U-I)^{-1}\right\|_{L^{p}(X) \rightarrow L^{p}(X)},
$$

and we obtain the desired result.

As a corollary from Theorem 2.2 and Theorem 3.1 we obtain the following characterization of relatively compact sets.

Theorem 3.4. Assume that $(X, \rho, \mu)$ is a connected metric measure space with continuous measure satisfying the doubling condition. Suppose, moreover, that balls are relatively compact and there exists $\theta>0$ such that $\mu(B(x, 1)) \geq \theta$. Let $1<p<$ $\infty$ and $D$ be a bounded subset of $X$ such that $X \backslash \bar{D} \neq \emptyset$. Then, the family $\mathcal{F}$ in $L^{p}(D, \mu)$ is relatively compact in $L^{p}(D, \mu)$ if and only if

$$
\lim _{r \rightarrow 0} \int_{X}\left|f(x)-(f)_{B(x, r)}\right|^{p} d \mu(x)=0 \quad \text { uniformly for } f \in \mathcal{F},
$$

where we continue the function $f$ by zero beyond $D$. 
Acknowledgements. H. R. was partially supported by Pontificia Universidad Javeriana under the research project "Study of non-standard Banach spaces", ID PPT: 6326.

\section{References}

[1] Gaczkowski, M., and P. GóRKA: Harmonic functions on metric measure spaces: convergence and compactness. - Potential Anal. 31, 2009, 203-214.

[2] Goes, S., and R. Welland: Compactness criteria for Köthe spaces. - Math. Ann. 188, 1970, 251-269.

[3] Górka, P.: Campanato theorem on metric measure spaces. - Ann. Acad. Sci. Fenn. Math. 34, 2009, 523-528.

[4] GóRkA, P.: Pego theorem on locally compact abelian groups. - J. Algebra Appl. 13:4, ID 1350143, 2014.

[5] Górka, P., and T. Kostrzewa: Pego everywhere. - J. Algebra Appl. 15:4, ID 1650074, 2016.

[6] Górka, P., and A. Macios: The Riesz-Kolmogorov theorem on metric spaces. - Miskolc Math. Notes 15, 2014, 459-465.

[7] Górka, P., and A. Macios: Almost everything you need to know about relatively compact sets in variable Lebesgue spaces. - J. Funct. Anal. 269:7, 2015, 1925-1949.

[8] Górka, P., and H. RAfeiro: From Arzelà-Ascoli to Riesz-Kolmogorov. - Nonlinear Anal. 144, 2016, 23-31.

[9] Hanche-Olsen, H., and H. Holden: The Kolmogorov-Riesz compactness theorem. - Expo. Math. 28, 2010, 385-394.

[10] Heinonen, J.: Lectures on analysis on metric spaces. - Universitext, 2001.

[11] Kolmogoroff, A.: Über die Kompaktheit der Funktionenmengen bei der Konvergenz im Mittle. - Nachr. Ges. Wiss. Göttingen, 1931, 60-63.

[12] Musielak, J.: Orlicz spaces and modular spaces. - Lecture Notes in Math. 1034, SpringerVerlag, Berlin, 1983.

[13] Pego, R. L.: Compactness in $L^{2}$ and the Fourier transform. - Proc. Amer. Math. Soc. 95, $1985,252-254$.

[14] Rafeiro, H.: Kolmogorov compactness criterion in variable exponent Lebesgue spaces. - Proc. A. Razmadze Math. Inst. 150, 2009, 105-113.

[15] Rafeiro, H., and A. Vargas: On the compactness in grand spaces. - Georgian Math. J. 22:1, $2015,141-152$.

[16] Sudakov, V. N.: Criteria of compactness in function spaces. - Uspehi Mat. Nauk (N.S.) 12:3(75), 1957, 221-224 (in Russian).

[17] Takahashi, T.: On the compactness of the function-set by the convergence in the mean of general type. - Studia Math. 5, 1934, 141-150.

[18] Tamarkin, J. D.: On the compactness of the space $L_{p}$. - Bull. Amer. Math. Soc 38,1932 , 79-84.

[19] Tulajkov, A.: Zur Kompaktheit im Raum $L_{p}$ für $p=1$. - Nachr. Gess. Wiss. Göttingen, Math. Phys. Kl, 1933, 167-170.

[20] WeIL, A.: L'intégration dans les groupes topologiques et ses applications. - Hermann, 1951.

[21] YosidA, K.: Functional analysis. - Springer-Verlag, Berlin-New York, 1980. 